\title{
Shock wave diffraction by a square cavity filled with dusty gas
}

\author{
B.Y. Wang ${ }^{1}$, Q.S. Wu ${ }^{1}$, C. Wang ${ }^{1}$, O. Igra ${ }^{2}$, J. Falcovitz ${ }^{3}$ \\ 1 Institute of Mechanics, Chinese Academy of Sciences, Beijing, P.R. China \\ 2 The Pearlstone Center for Aeronautical Studies, Department of Mechanical Engineering, \\ Ben-Gurion University of the Negev, Beer Sheva, Israel \\ 3 Institute of Mathematics, Hebrew University, Jerusalem, Israel
}

Received 15 November 1999 / Accepted 25 October 2000

\begin{abstract}
A simple two-dimensional square cavity model is used to study shock attenuating effects of dust suspension in air. The GRP scheme for compressible flows was extended to simulate the fluid dynamics of dilute dust suspensions, employing the conventional two-phase approximation. A planar shock of constant intensity propagated in pure air over flat ground and diffracted into a square cavity filled with a dusty quiescent suspension. Shock intensities were $M_{s}=1.30$ and $M_{s}=2.032$, dust loading ratios were $\alpha=1$ and $\alpha=5$, and particle diameters were $d=1,10$ and $50 \mu \mathrm{m}$. It was found that the diffraction patterns in the cavity were decisively attenuated by the dust suspension, particularly for the higher loading ratio. The particle size has a pronounced effect on the flow and wave pattern developed inside the cavity. Wall pressure histories were recorded for each of the three cavity walls, showing a clear attenuating effect of the dust suspension.
\end{abstract}

Key words: Shock wave attenuation, Dusty gas dynamics, Shock wave diffraction

\section{Introduction}

Shock wave propagation, diffraction and reflection phenomena in dusty air suspensions were the subject of numerous experimental and theoretical studies in recent decades. Most of the early works were numerical studies, treating one dimensional (planar), quasi-steady flow fields, e.g., the pioneering studies of Carrier (1958), Rudinger (1964) and Kriebel (1964). These simple models were extended to cover spherical dusty shock wave flows, e.g., Gerber and Barton (1974) and Igra, Elperin and Ben-Dor (1987) and truly unsteady, planar, one-dimensional shock wave propagation into dusty gas; for example Marconi, Rudman and Calia (1981) and Miura \& Glass (1985). A frequently employed assumption in almost all numerical investigations is that the dust is uniformly distributed in the gas. This is hard to achieve in reality and as a result the number of published experimental studies is much smaller than the published numerical studies. Among the available information one should mention the work of Sommerfeld (1985), that by Sugiyama, Arai, Nagumo, Sueki, Izumi and Takayama (1989) and the work of Geng and Grönig (2000). The present study is a numerical simulation of a simplified configuration in this class of shock wave phenomena. An understanding of these phenomena is needed in the analysis of accidents related to public safety, such as dust explosions in silos, explosions in mines

Correspondence to: Ozer Igra (e-mail: ozer@bgumail.bgu.ac.il) or in petrochemical industry, as well as numerous other instances of industrial accidents that produce an air blast. Following such explosions, the effects of the air blast are typically transmitted to large distance via shock wave propagation. A complex flow is generated behind the shock front due to multiple interactions between the transmitted shock wave and structures encountered by the shock.

The presence of solid particles suspended in the air may considerably affect the shock wave propagation and its interaction pattern with encountered obstacles. Consequently, blast load histories depend on the strength of the incident shock wave, the obstacle geometry, the particle size and its physical properties, and the particles spatial density (dust mass loading ratio).

The model configuration chosen for this study is a two-dimensional square cavity embedded in a flat surface and filled with a uniform dust-air suspension. The entire medium is initially quiescent, except for an incident planar shock that propagates in the pure air above the surface, with its front normal to the surface. The interaction under study starts when the shock arrives at the leading edge of the square cavity, and diffracts into the cavity through its open top face. The suspension is modeled as a perfect gas seeded with tiny identical particles uniformly distributed throughout the cavity. As is known from previous numerical and experimental studies in pure air (e.g., see Igra et al. 1996), the shock-induced cavity flow involves diffracted 
shock waves, reflected shock waves, expansion waves, as well as shear layer and vortex regions accompanied by pressure-matching shocks. This array of complex shock wave phenomena will obviously be affected by the particles suspended in the cavity, to an extent commensurate with the dust mass loading ratio and the other physical characteristics of the particles.

\section{Model formulation}

In the present study, a two-fluid model is employed for simulating the gas-particle suspension, where the particle phase is dilute enough to ignore its partial volume and its partial pressure in the suspension. The particles are assumed to be solid rigid spheres, their sole physical interaction with the gas being an exchange of momentum and of heat. Assuming that the carrying gas is inviscid yields that the flow governing equations are the Euler equations expressing conservation of mass, momentum and energy. It is also assumed that the considered compressible gas can be treated as an ideal gas, i.e., it has a simple equation of state. Likewise, the "particle fluid" flow is also governed by the hydrodynamic conservation laws, but for a fluid having zero-pressure. Under these assumptions the basic equations for the considered nonstationary two-phase flow can be written in the Cartesian coordinates $(x, y)$ as:

$$
\frac{\partial U}{\partial t}+\frac{\partial F}{\partial x}+\frac{\partial G}{\partial y}=H
$$

where $U$ is the vector of unknown flow variables; $F, G$ and $H$ are the fluxes in the $x$ - and $y$-directions and the source terms, which are as follows

$$
\begin{gathered}
U=\left(\begin{array}{l}
\rho_{g} \\
\rho_{g} u_{g} \\
\rho_{g} v_{g} \\
\rho_{g} E_{g} \\
\rho_{p} \\
\rho_{p} u_{p} \\
\rho_{p} v_{p} \\
\rho_{p} E_{p}
\end{array}\right), \quad F=\left(\begin{array}{l}
\rho_{g} u_{g} \\
\rho_{g} u_{g}^{2}+p \\
\rho_{g} u_{g} v_{g} \\
u_{g}\left(\rho_{g} E_{g}+p\right) \\
\rho_{p} u_{p} \\
\rho_{p} u_{p}^{2} \\
\rho_{p} u_{p} v_{p} \\
\rho_{p} u_{p} E_{p}
\end{array}\right) \\
G=\left(\begin{array}{l}
\rho_{g} v_{g} \\
\rho_{g} u_{g} v_{g} \\
\rho_{g} v_{g}^{2}+p \\
v_{g}\left(\rho_{g} E_{g}+p\right) \\
\rho_{p} v_{p} \\
\rho_{p} u_{p} v_{p} \\
\rho_{p} v_{p}^{2} \\
\rho_{p} v_{p} E_{p}
\end{array}\right), \quad H=\left(\begin{array}{l}
0 \\
-f_{x} \\
-f_{y} \\
-u_{p} f_{x}-v_{p} f_{y}-q \\
0 \\
f_{x} \\
f_{y} \\
u_{p} f_{x}+v_{p} f_{y}+q
\end{array}\right) .
\end{gathered}
$$

The gas is assumed to obey a polytropic equation of state:

$$
p=\rho_{g} R T_{g} .
$$

In the equations above, variables $p, \rho, T$ and $u, v$ are the pressure, density, temperature and velocity components in the $x$ - and $y$-directions (here the subscripts $g$ and $p$ refer to the gas and particles respectively); $E=$ $C_{m} T+\left(u^{2}+v^{2}\right) / 2$ is the specific total energy $\left(C_{v}\right.$ is the specific heat capacity of the gas at constant volume and $C_{m}$ is the heat capacity of particle material). The source terms in the right side of Eq. (1) create a coupling between the equations governing the flow of the two phases. The viscous interaction between the gas and particles is accounted for by the drag force $\vec{f}$ and heat exchange rate $q$ :

$$
\begin{aligned}
f_{x} & =\frac{\pi d^{2}}{8 m} \rho_{p} \rho_{g}\left(u_{g}-u_{p}\right) \sqrt{\left(u_{g}-u_{p}\right)^{2}+\left(v_{g}-v_{p}\right)^{2}} C_{D} \\
f_{y} & =\frac{\pi d^{2}}{8 m} \rho_{p} \rho_{g}\left(v_{g}-v_{p}\right) \sqrt{\left(u_{g}-u_{p}\right)^{2}+\left(v_{g}-v_{p}\right)^{2}} C_{D} \\
q & =\frac{\pi d}{m} \cdot \rho_{p} \frac{\mu C_{p}}{P_{r}}\left(T_{g}-T_{p}\right) N_{u}
\end{aligned}
$$

where $m$ and $d$ are the mass and diameter of a single particle; $\mu, \operatorname{Pr}$ and $C_{p}$ are the gas viscosity, Prandtl number and specific heat capacity at constant pressure. In addition, empirical expressions for the drag coefficient $C_{D}$ and Nusselt number $N u$ are chosen from the available literature. Frequently used expressions are (Igra and Ben-Dor, 1988):

$$
\begin{aligned}
C_{D} & =0.48+28 \operatorname{Re}^{-0.85} \\
N u & =2.0+0.6 \operatorname{Pr}^{1 / 3} \operatorname{Re}^{1 / 2} \\
R e_{s} & =\rho_{g}\left[\left(u_{g}-u_{p}\right)^{2}+\left(v_{g}-v_{p}\right)^{2}\right]^{1 / 2} d / \mu
\end{aligned}
$$

where $R e_{s}$ is the slip Reynolds number based on the particle diameter. These expressions for $C_{D}$ and $N u$ were also employed in Rudinger and Chang (1964), Miura \& Glass (1985), Igra, Elperin and Ben-Dor (1987) and many others. Use of similar correlations for $C_{D}$ and $N u$ in gasdynamic conservation laws schemes has also been validated by comparison to experiments, e.g., Aizik et al. (1995).

\section{Numerical schemes}

Using the operator-splitting technique, Eq. (1) is replaced by the following three conservation laws:

$$
\begin{gathered}
\frac{\partial U}{\partial t}+\frac{\partial F}{\partial x}=0 \\
\frac{\partial U}{\partial t}+\frac{\partial G}{\partial y}=0 \\
\frac{d U}{d t}=H .
\end{gathered}
$$




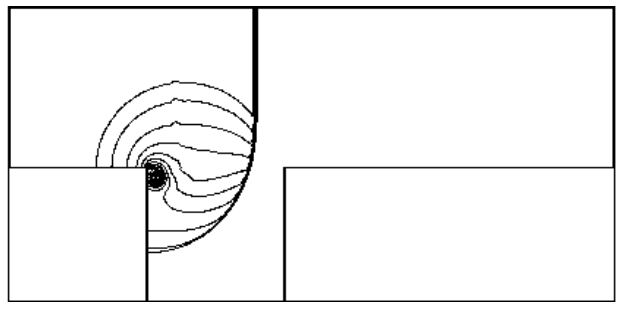

a $t=100 \mu \mathrm{s}$

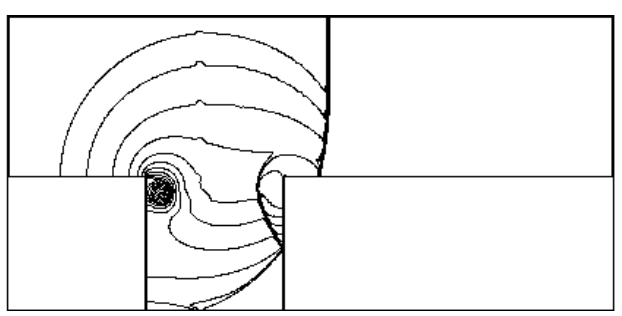

b $t=160 \mu \mathrm{s}$

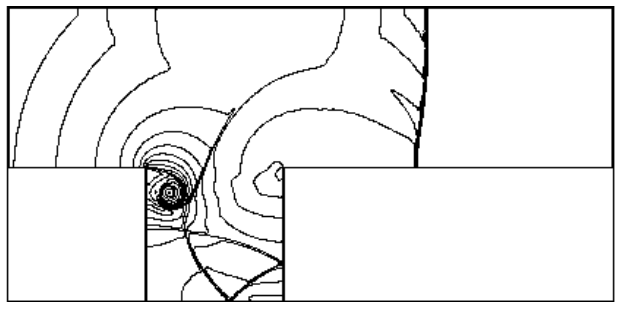

c $t=240 \mu \mathrm{s}$

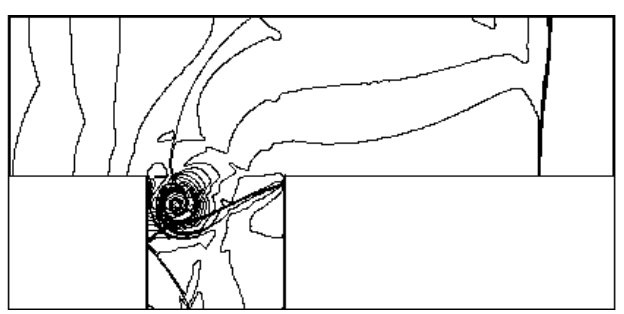

$\mathbf{d} t=240 \mu \mathrm{s}$

Fig. 1a-d. Wave pattern for $M_{s}=1.30, \alpha=1$ and $d=10 \mu \mathrm{m}$

Due to the split procedure, the two-dimensional finitedifference scheme for the integration of Eq. (1) can be obtained by applying a sequence of one-dimensional conservation law schemes to Eq. (3). Moreover, in Eqs. (3a,b), the gas-phase equation is not coupled with the particlephase equation. The gas Euler equation in conservative form is solved using the GRP scheme (Ben-Artzi and Falcovitz 1984, 1986; Falcovitz and Ben-Artzi 1995). The proposed numerical scheme GRP (Generalized Riemann Problem) is especially suitable for producing solutions to compressible flows with shock or contact discontinuities, which characterize shock wave diffraction phenomena. The particle fluid of zero-pressure, however, require a different numerical method; for example the MacCormack scheme (MacCormack 1969) for solving the particle-phase equation. Let $L_{x}$ and $L_{y}$ be the one-dimensional operators corresponding to Eqs. (3a) and (3b), the integration of

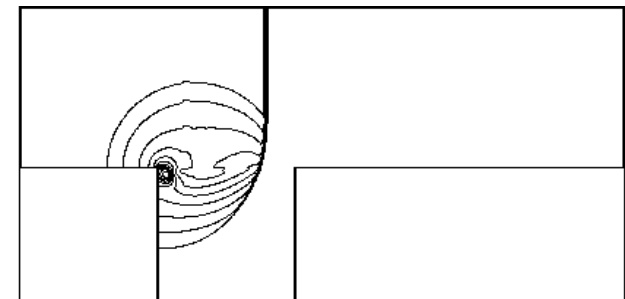

a $t=100 \mu \mathrm{s}$

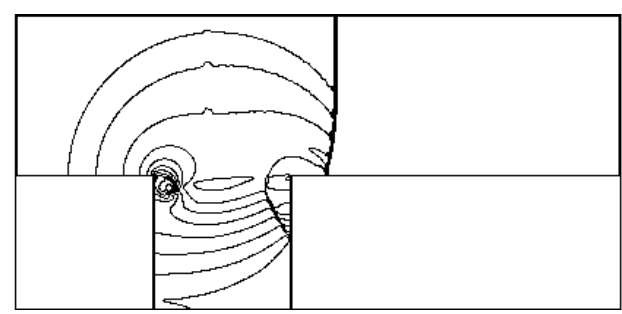

b $t=160 \mu \mathrm{s}$

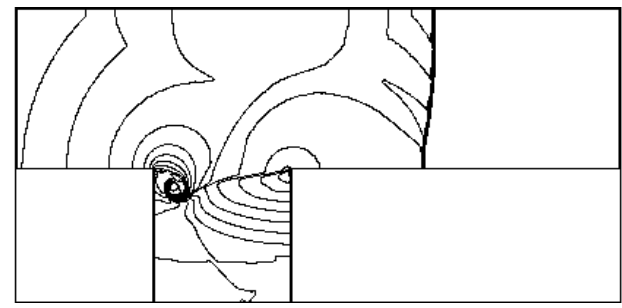

c $t=240 \mu \mathrm{s}$

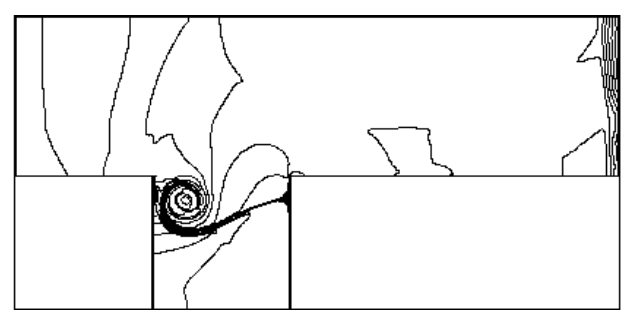

$\mathbf{d} t=340 \mu \mathrm{s}$

Fig. 2a-d. Wave pattern for $M_{s}=1.30, \alpha=5$ and $d=10 \mu \mathrm{m}$

Eqs. (3a,b) for the gas and particles are given, respectively, by:

$$
\begin{gathered}
U_{g}^{n+1}=L_{x}(\Delta t) L_{y}(\Delta t) U_{g}^{n} \\
U_{p}^{n+1}=L_{x}\left(\frac{\Delta t}{2}\right) L_{y}(\Delta t) L_{x}\left(\frac{\Delta t}{2}\right) U_{p}^{n} .
\end{gathered}
$$

Here, based on the observation in Igra et al. (1996), the simplified splitting given in Eq. (4) is adopted since it is more efficient and yields virtually indistinguishable results compared with the more accurate splitting given by Strang (1968). Equation (3c) is solved using the predictorcorrector scheme and for each half time-step, its integration is obtained using a predictor step followed by a corrector step

$$
U_{i, j}^{(1)}=U_{i, j}^{n}+\frac{\Delta t}{2} H_{i, j}^{n}
$$




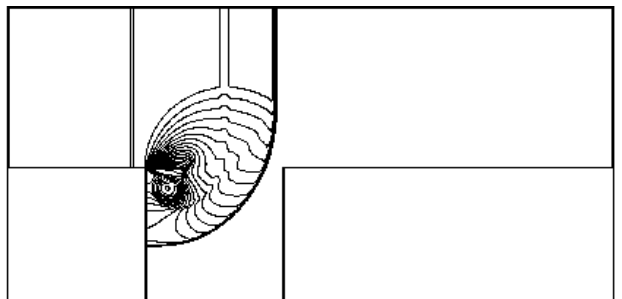

a $t=75 \mu \mathrm{s}$

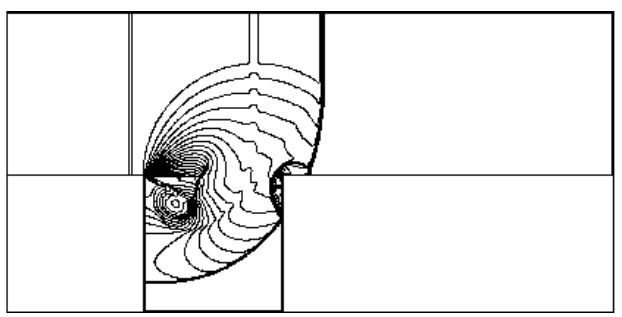

b $t=100 \mu \mathrm{s}$

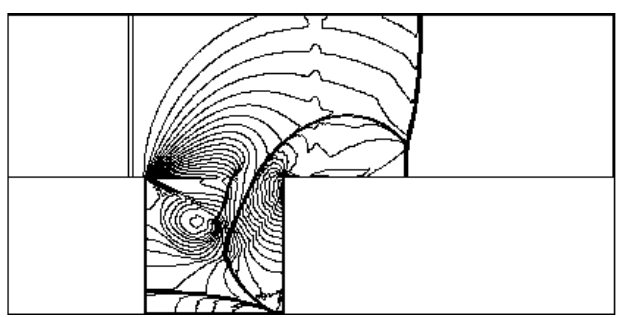

c $t=150 \mu \mathrm{s}$

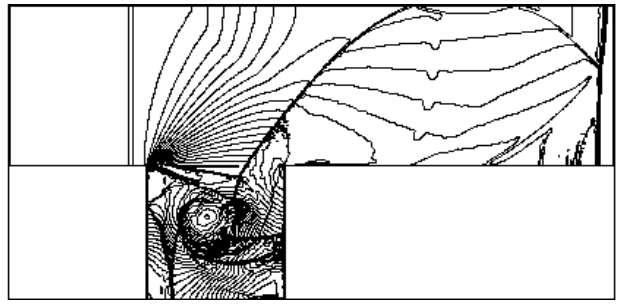

$\mathbf{d} t=250 \mu \mathrm{s}$

Fig. 3a-d. Wave pattern for $M_{s}=2.032, \alpha=1$ and $d=10 \mu \mathrm{m}$

$$
U_{i, j}^{n+1 / 2}=U_{i, j}^{n}+\frac{\Delta t}{4}\left(H_{i, j}^{n}+H_{i, j}^{(1)}\right) .
$$

All the above schemes are finite-difference schemes of second-order accuracy in time and in space.

The computational domain is a rectangle of $220 \times$ $110 \mathrm{~mm}$, and is covered by a uniform Cartesian mesh composed of $660 \times 330$ square cells. The rectangle is divided into two parts by a horizontal line $50 \mathrm{~mm}$ above the bottom, representing a flat ground surface. The square $(50 \times$ $50 \mathrm{~mm}$ ) cavity is embedded in that surface, with its bottom face coinciding with that of the rectangle. As mentioned earlier, there is pure gas (air) above the ground surface and dusty gas inside the square cavity.

A "physical" validation, aimed at the validity and accuracy of the two-phase modeling has been conducted with respect to shock tube experiments where a planar shock wave has been driven into a dusty gas suspension

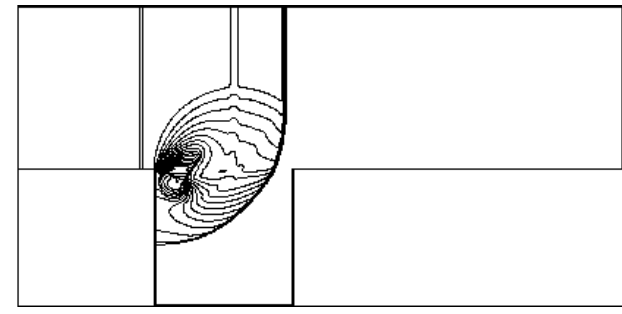

a $t=75 \mu \mathrm{s}$

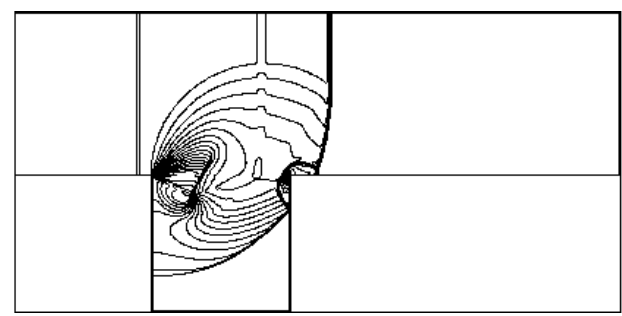

b $t=100 \mu \mathrm{s}$

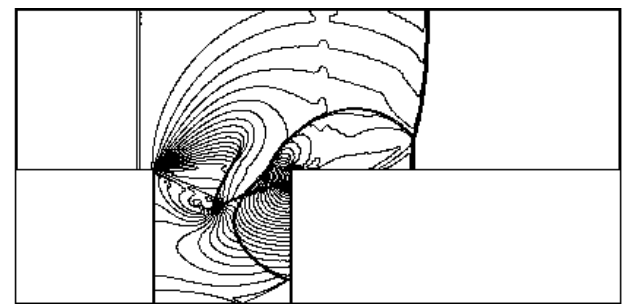

c $t=150 \mu \mathrm{s}$

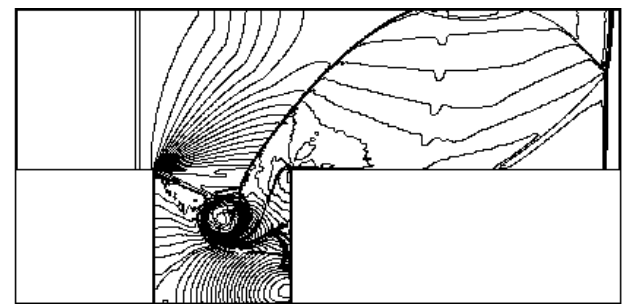

$\mathbf{d} t=250 \mu \mathrm{s}$

Fig. 4a-d. Wave pattern for $M_{s}=2.032, \alpha=5$ and $d=10 \mu \mathrm{m}$

(Sommerfeld 1985). Good agreement was observed between the measured shock wave attenuation and the appropriate simulations.

\section{Results and discussion}

Numerical simulations of shock wave diffraction into a square cavity filled with a gas-solid particles suspension were performed for the following twelve cases. Two different values of the incident shock wave Mach number, $M_{s}=1.30$ and $M_{s}=2.032$; for each of the incident shock waves the solution was obtained for two different dust loadings ( $\alpha=1$ and 5) and three different particle diameters $(d=1,10$ and $50 \mu \mathrm{m})$. In all cases the dust particles had a material density of $2500 \mathrm{~kg} / \mathrm{m}^{3}$. The resulting post-shock flow and wave patterns are shown in Figs. 1-4 for different times during the flow duration. Lines shown in these figures are lines of constant density (isopycnics) 


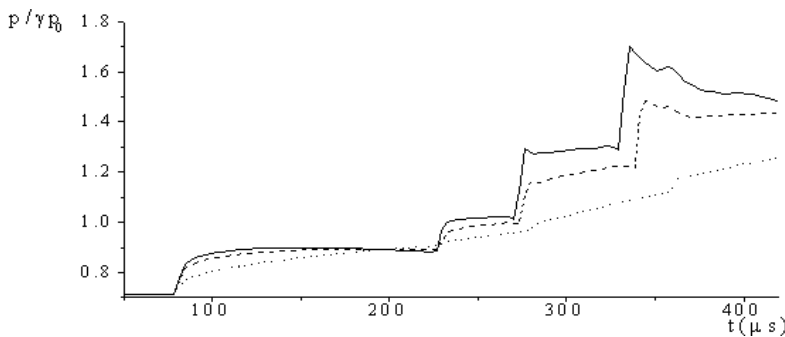

a at midpoint on left wall

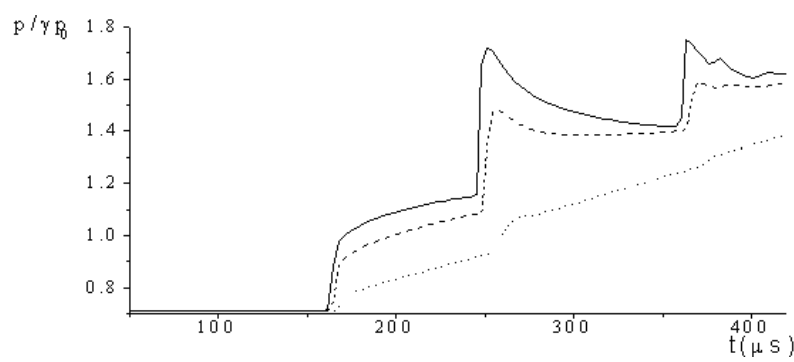

$\mathbf{b}$ at midpoint on bottom wall

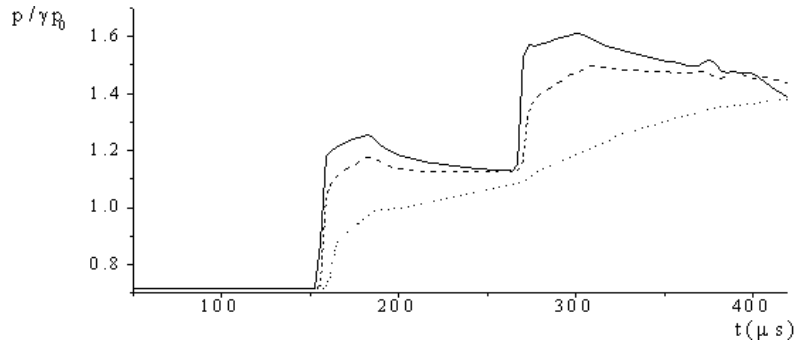

c at midpoint on right wall

Fig. 5a-c. Time history of pressure for $M_{s}=1.30$ (solid line: $\alpha=0$; dash line: $\alpha=1$; dot line: $\alpha=5$ )

in the gaseous phase. For visualization of the dust effect on the resulting flow the reader should compare Figs. 14 with the corresponding dust-free results given in Igra et al. (1996). Results shown in Figs. 1-4 indicate that the wave pattern developed inside and around the cavity is controlled by multiple wave interactions with the cavity walls and among the various shock waves present in the considered flow field. Comparing Fig. $1(\alpha=1)$ with Fig. $2(\alpha=5)$ indicates that changes in the dust loading do not affect the strength of the transmitted shock wave, i.e., it propagates with nearly the same velocity for both loadings. However, the presence of solid particles in the gaseous phase inside the cavity significantly affects the propagation of the diffracted and the reflected shock waves inside the cavity and the subsequent shock interactions. Increasing the dust load results in weakening the shock wave's strength (lowering its velocity) inside the cavity. For the relatively high dust loading $(\alpha=5)$ shock waves inside the cavity decay to compression waves. In Figs. 3 and 4 the incident shock wave is stronger than that of the previous case; it is $M_{s}=2.032$. As a result the post shock flow is almost sonic and the flow expands into the cavity via a centered expansion wave, rather than through a vortex as was seen in Figs. 1 and 2 (which is typical of sub-

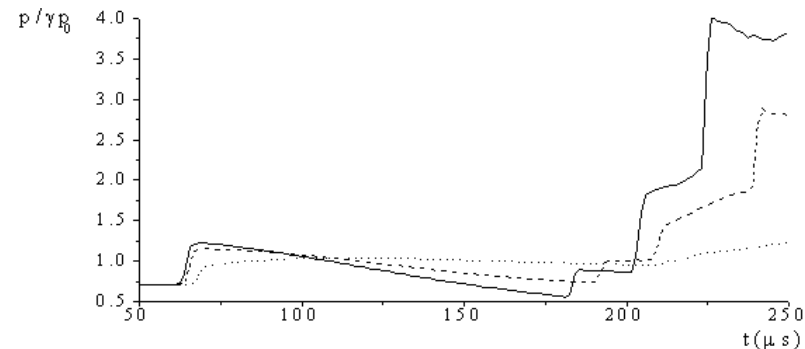

a at midpoint on left wall

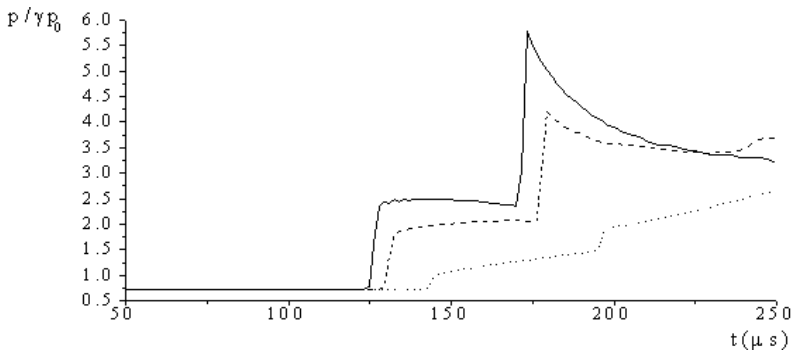

b at midpoint on bottom wall

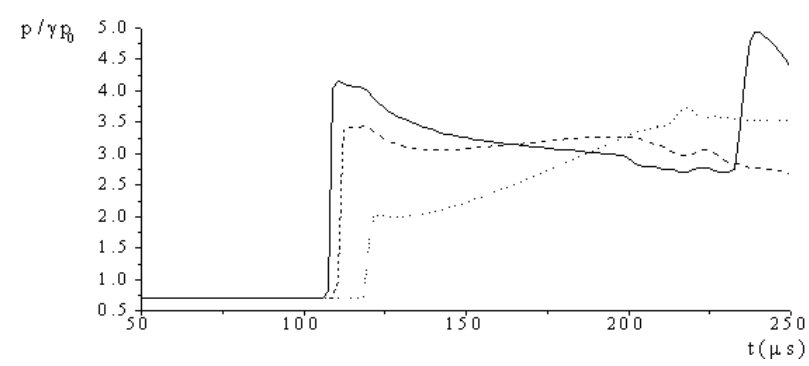

c at midpoint on right wall

Fig. 6a-c. Time history of pressure for $M_{s}=2.032$ (solid line: $\alpha=0$; dash line: $\alpha=1$; dot line: $\alpha=5$ )

sonic flow expansion). In the present case (Figs. 3 and 4) a secondary upstream facing shock wave is present inside the cavity, just behind the expansion fan. Its presence is necessary for matching the low pressure produced by the expansion wave, centered at the cavity upper left corner, to the relatively high pressure prevailing behind the transmitted shock wave. Similar to what was observed in the previous case, here too the dust presence inside the cavity has practically no effect on the incident shock wave passage over the cavity. It affects the flow and wave pattern developed inside, and around, the cavity; increasing the dust loading leads to a decrease in the strength of all shock waves inside the cavity. However, since the incident shock wave in the present case $\left(M_{s}=2.032\right)$ is stronger than that of the previous case $\left(M_{s}=1.30\right)$, not all shock waves now decay to compression waves. Pressure histories (normalized by the pre-shock pressure, $p_{0}$ ) prevailing at the center of each of the cavity's walls are given in Figs. 5 and 6 for $M_{s}=1.30$ and $M_{s}=2.032$, respectively. Each figure has three curves indicating the following dust loadings: $\alpha=0,1$, and 5. From these curves the effect of dust loading on the obtained pressures can easily be deduced. It is clear from Figs. 5 and 6 that the strongest shock waves are experienced in the dust-free case. The 


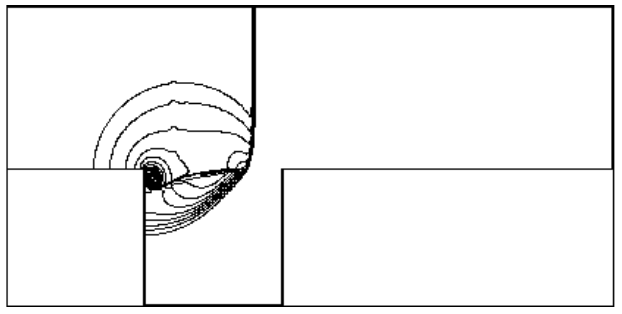

a $t=100 \mu \mathrm{s}$

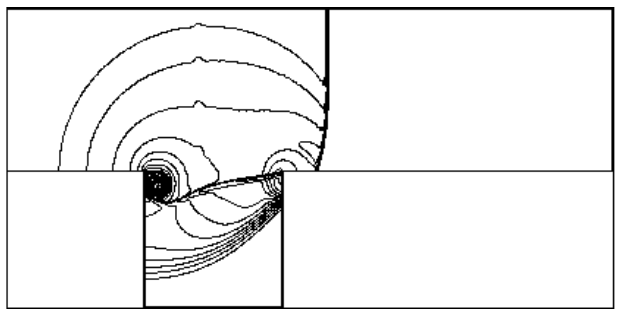

b $t=160 \mu \mathrm{s}$

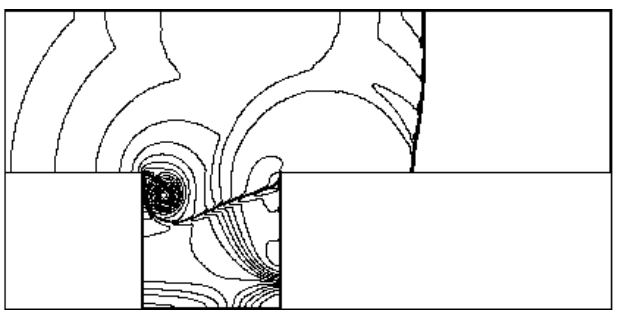

c $t=240 \mu \mathrm{s}$

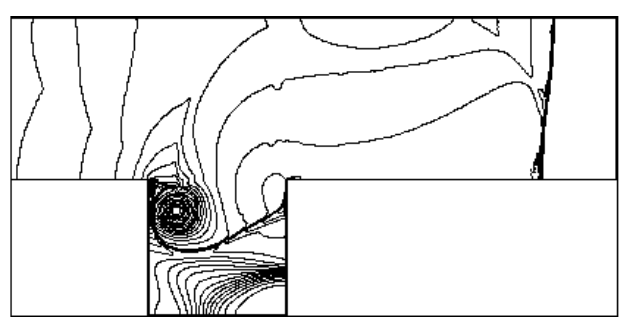

$\mathbf{d} t=340 \mu \mathrm{s}$

Fig. 7a-d. Wave pattern for $M_{s}=1.30, \alpha=1$ and $d=1 \mu \mathrm{m}$

dust presence inside the cavity results in attenuation of the diffracted and reflected shock waves. The higher the dust loading, the weaker are the shock waves. As could be expected, the highest pressures are observed on the right wall of the cavity, where the incident shock wave hits the wall head-on. The lowest pressures are experienced on the left wall of the cavity, over which the incident shock wave diffracts. For the case of a relatively weak incident shock wave $\left(M_{s}=1.30\right.$ shown in Fig. 5$)$ the dust presence, especially in the high dust loading case, reduces some of the shock waves to compression waves. The effect of shock wave weakening due to the dust presence is also noticeable in Fig. 6 where $M_{s}=2.032$. However, for the time duration covered in this figure waves appearing in the suspension are still shock waves, not compression waves. For the case of a relatively high dust loading ( $\alpha=5$, Fig. 6$)$ an interesting phenomenon is evident. While the observed pressure jump through the incident and the reflected shock

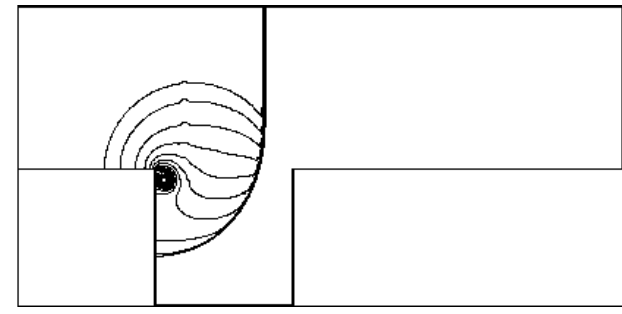

a $t=100 \mu \mathrm{s}$

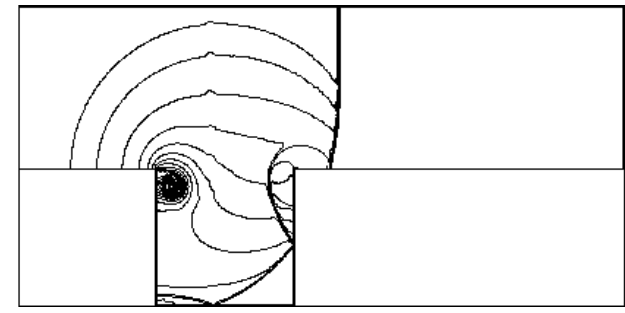

b $t=160 \mu \mathrm{s}$

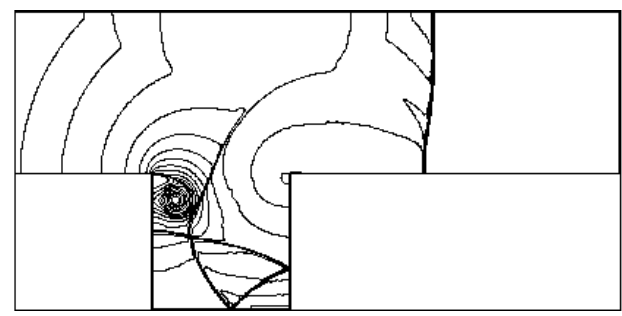

c $t=240 \mu \mathrm{s}$

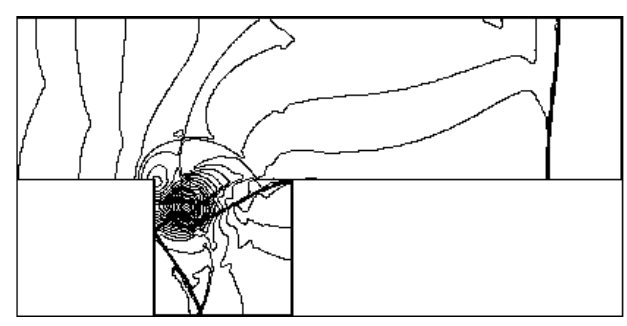

d $t=340 \mu \mathrm{s}$

Fig. 8a-d. Wave pattern for $M_{s}=1.30, \alpha=1$ and $d=50 \mu \mathrm{m}$

waves are smaller than those obtained in a similar pure gas case, in the suspension case pressures continue to increase behind the wave's front. This is in contrast to a similar pure gas case where pressure decrease is normally encountered behind the shock front. The shock wave attenuation due to the dust presence inside the square cavity can also be clearly visible from the time it takes until the incident, and the reflected, shock waves reach the mid point of the cavity's right (or bottom) wall; see Fig. 6 b and $6 \mathrm{c}$.

In the present numerical solution, the effects associated with changes made in the solid particles diameter on the resulting flow were also studied. The obtained results are shown in Figs. 7-10. As could be expected, due to the large difference in inertia between the two sets of particles $(1$, and $50 \mu \mathrm{m})$, the small particles reach equilibrium with the carrying gas very quickly and thereby affect the resulting flow field. This is clearly noticed in Fig. 7 where all the shock waves which were observed in the pure gas case (see 


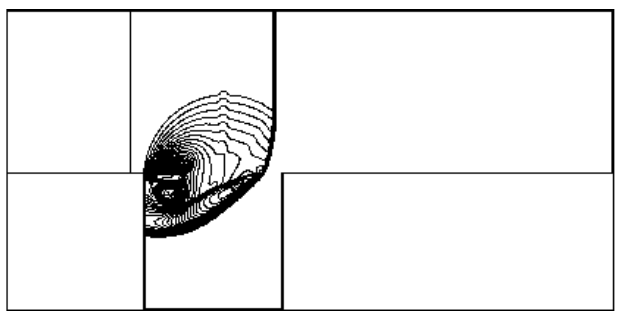

a $t=75 \mu \mathrm{s}$

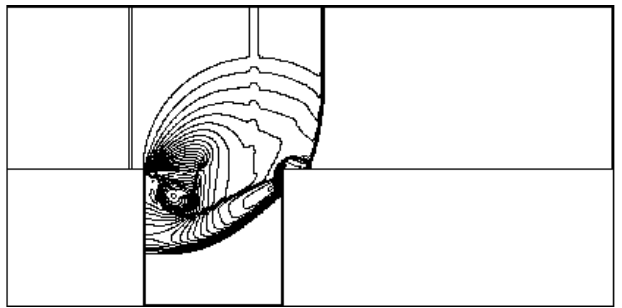

b $t=100 \mu \mathrm{s}$

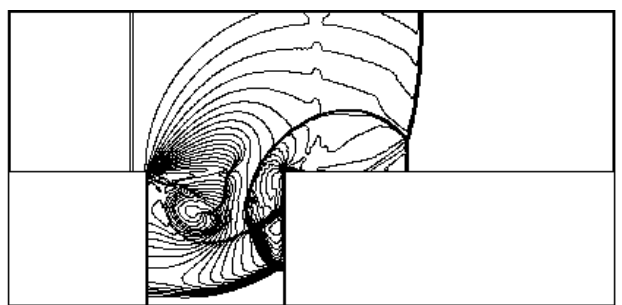

c $t=150 \mu \mathrm{s}$

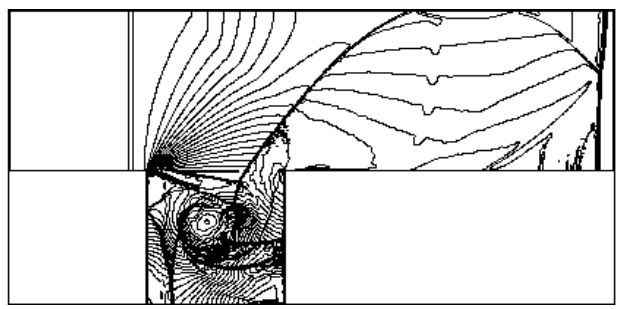

d $t=250 \mu \mathrm{s}$

Fig. 9a-d. Wave pattern for $M_{s}=2.032, \alpha=1$ and $d=1 \mu \mathrm{m}$

in Igra et al. 1996) are significantly weakened now and eventually reduce to compression or Mach waves. On the other hand, the large particles $(\mathrm{d}=50 \mu \mathrm{m})$ require a much longer time for reaching equilibrium with the carrying gas. Therefore, for the time period covered in the present computations the flow is practically frozen as is apparent from comparing the results shown in Fig. 8 with those of the pure gas case, (Igra et al. 1996). The wave pattern shown in Fig. 8 is very different from that shown in Fig. 7. A similar, but not identical, behaviour is observed in the case of a stronger incident shock wave shown in Figs. 9 and 10. Here too, the small particles $(d=1 \mu \mathrm{m}$ in Fig. 9$)$ interact quickly with the gaseous phase to result in significant attenuation of all waves, excluding the leading, incident shock wave. When comparing the wave pattern observed in Figs. 9 and 10 at relatively short times after the incident shock wave entered the square cavity, i.e., $0<t<150 \mu \mathrm{s}$, it is clearly seen that significant wave attenuation is ex-

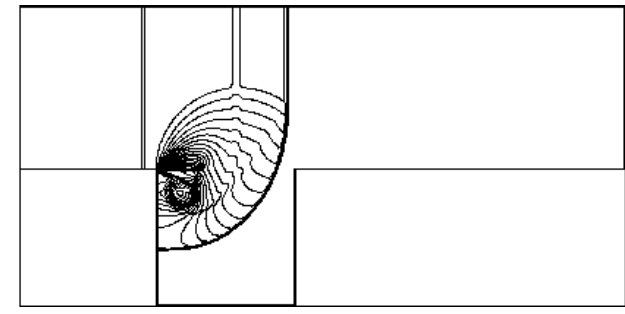

a $t=75 \mu \mathrm{s}$

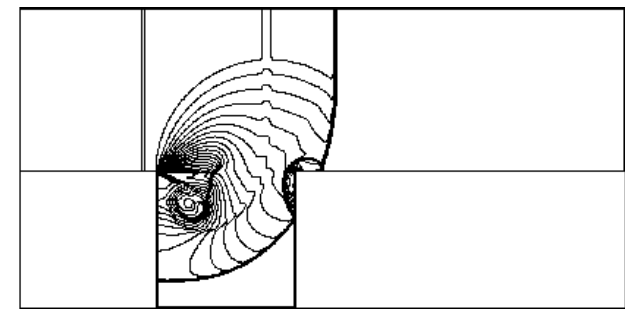

b $t=100 \mu \mathrm{s}$

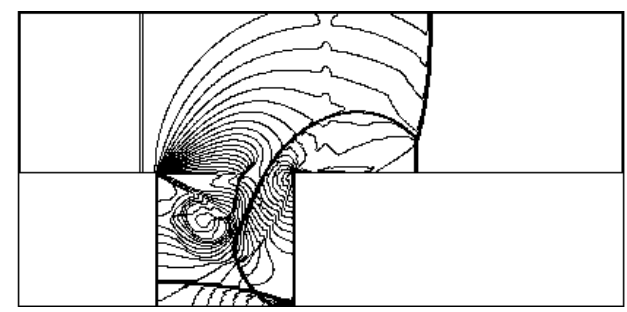

c $t=150 \mu \mathrm{s}$

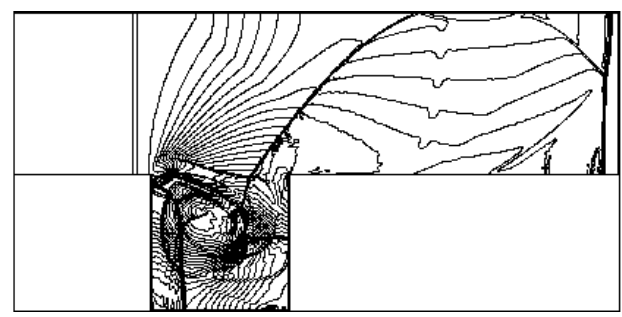

d $t=250 \mu \mathrm{s}$

Fig. 10a-d. Wave pattern for $M_{s}=2.032, \alpha=1$ and $d=$ $50 \mu \mathrm{m}$

perienced by the suspension composed of small particles $(d=1 \mu \mathrm{m})$. While the wave pattern observed in Fig. 10 is different from that of Fig. 9, it is very similar to that observed in a similar pure-gas case (Igra et al. 1996). This is an indication that the suspension composed of large particles $(d=50 \mu \mathrm{m})$ behaves as a frozen flow. All waves, excluding the incident shock wave, in the small particles suspension (Fig. 9) are much slower than their counterparts in the large particles suspension (Fig. 10). At late times, $t \geq 250 \mu \mathrm{s}$, heat and momentum transfer between the two phases of the suspension composed of large particles $(d=50 \mu \mathrm{m}$ in Fig. 10$)$ is activated to result in a similar wave pattern between the two suspensions; see Figs. 9d and 10d. However, the observed wave pattern is still not identical in both figures. It will take a longer time until a real similar wave pattern will be observed in both suspensions. 


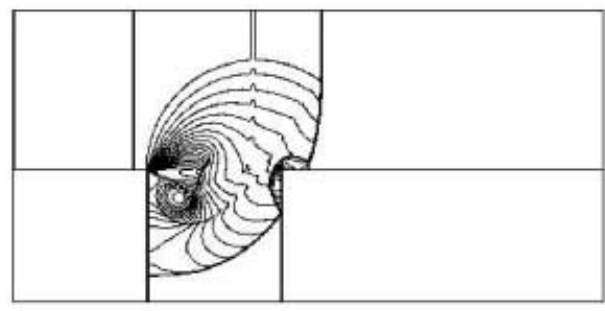

a $t=100 \mu \mathrm{s}$

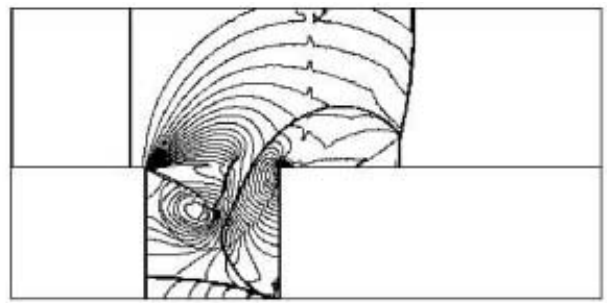

b $t=150 \mu \mathrm{s}$

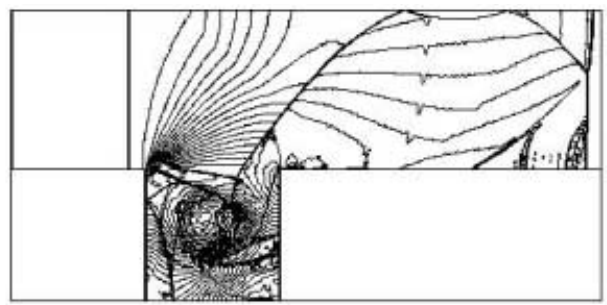

c $t=250 \mu \mathrm{s}$

Fig. 11a-c. As in Fig. 3 but with doubling the number of the grid points

In order to remove any doubt as to whether or not the results presented here are mesh dependent, we repeated the case shown in Fig. 3, but now with a mesh having twice the number of points in each direction. The obtained results, shown in Fig. 11, clearly exhibit that doubling the number of grid points did not alter the obtained wave pattern and isopycnics.

\section{Conclusions}

The wave diffraction resulting from the interaction between a planar shock wave and a square cavity filled with dusty gas was studied in detail. It was shown that an array of complex wave phenomena in two-phase media could be simulated numerically by using the second-order GRP and MacCormack schemes. Based on the obtained numerical results, the following is concluded:

1. No observable changes are found for the flow above the cavity, and the passing shock wave is almost unaffected by the mass loading ratio of the gas-particle suspension inside the cavity.

2. The presence of solid particles inside the cavity brings about significant changes in the cavity flow relative to a similar pure gas case. The speed of diffracted and reflected shock waves decreases as the dust mass loading ratio increases, or the dust particle diameter decreases.
3. When the mass loading ratio is high enough or the particle diameter is small enough, all shock waves inside the square cavity become fully dispersed, i.e., they turn into compression waves.

4. The level of dust loading has a significant influence on the pressure acting on the cavity walls.

Acknowledgement. This work was supported by The Natural Science Foundation of China.

\section{References}

Aizik F, Ben-Dor G, Elperin T, Igra O and Mond M (1995) Attenuation law of planar shock waves propagating through dust-gas suspensions. AIAA J. 33: 953-955

Ben-Artzi M and Falcovitz J (1984) A Second-Order GodunovType Scheme for Compressible Fluid Dynamics. J. Comput. Phys. 55: 1-32

Ben-Artzi M and Falcovitz J (1986) An Upwind Second-Order Scheme for Compressible Duct Flows. SIAM J. Sci. Statist. Comp. 7: 744-768

Carrier GF (1958) Shock waves in a dusty gas. J Fluid Mech. 4: $376-382$.

Falcovitz J and Ben-Artzi M (1995) Recent Developments of the GRP method. JSME Intl. J B 38: 497-517

Geng JH and Grönig H (2000) Dust suspensions accelerated by shock waves. Experiments in Fluids 28: 360-367

Gerber N and Barton JM (1974) Strong spherical blast waves in a dust-laden gas. AIAA J 12: 120-122

Igra O and Ben-Dor G (1988) Dusty shock waves. Appl. Mech. Rev. 41: 379-437

Igra O, Elperin T and Ben-Dor J (1987) Blast waves in dusty gases. Proc. R. Soc. Lond. A414: 197-219

Igra O, Falcovitz J, Reichenbach H and Heilig W (1996) Experimental and Numerical Study of the Interaction between a Planar Shock Wave and a Square Cavity. J. Fluid Mech. 313: $105-130$

Kriebel, AR (1964) Analysis of normal shock waves in a particle-laden gas. Trans. ASME J Basic Eng. 86: 655-665

MacCormack RW (1969) The Effect of Viscosity on Hypervelocity Impact Cratering. AIAA Paper 69-354

Marconi F, Rudman S and Calia V (1981) Numerical study of one-dimensional unsteady particle-laden flows with shocks. AIAA J 19: 1294-1301

Miura H and Glass II (1985) On the passage of a shock wave through a dusty-gas layer. Proc. R. Soc. Lond. A 385: 85105

Rudinger G (1964) Some properties of shock relaxation in gas flows carrying small particles. Phys. Fluids 7: 1747-1754

Rudinger G and Chang A (1964) Analysis of nonsteady twophase flow. Phys. Fluids 7: 658-663

Sommerfeld M (1985) The unsteadiness of shock waves propagating through gas-particle mixtures. Experiments in Fluids 3: $197-206$

Strang G (1968) On the Construction and Comparison of Difference Schemes. SIAM J. Numer. Anal. 5: 506-517

Sugiyama H, Arai T, Nagumo H, Sueki H, Izumi $M$ and Takayama K (1989) An experimental and numerical study of the shock wave-induced flows past a circular cylinder in a dusty-gas shock tube. In Proceedings of the $17^{\text {th }}$ International Symposium on Shock Waves and Shock Tubes, Bethlehem, Pannsylvania, USA, 1989 